in sporadic episodes and pointers from school outbreaks have suggested that the incubation period of winter vomiting disease is usually 30 to 60 hours, though it may be as long as a week. ${ }^{8}$

Two other forms of a similar or of the identical condition have been reported in Britain. Firstly, the condition known as epidemic vertigo-which some workers think is an acute labyrinthitis but in which vomiting may be a prominent symptom-can scarcely be distinguished on clinical grounds from winter vomiting disease, in which vertigo is commonly present. In epidemic vertigo the symptoms are usually very sudden in onset. The patient is seized without warning with severe vertigo and either falls to the ground or staggers and sways uncontrollably. In some outbreaks of epidemic vertigo obvious involvement of the oculomotor nerve has been reported..$^{13}$ The second form has been described as epidemic collapse. In a large outbreak of this type in a Coventry girls' school the onset was sudden, with frontal headache, nausea, violent shivering, colicky upper abdominal pain, giddiness, and faintness. ${ }^{10}$ Reports in the press of the recent Blackburn outbreak indicate a clinical picture that is very similar to that seen at Coventry. Whatever the similarity, it is now admitted "that mass hysteria is the probable explanation of the Blackburn episode."14 In this connexion Sir Francis Walshe's comment in a letter to The Times of 19 October is pertinent: "Epidemics of mass hysteria have punctuated history and doubtless a few centuries ago were attributed to witchcraft or to demoniacal possession on evidence not less than that now evoking the mystic chant of the word "virus.",

With the large explosive outbreak of winter vomiting disease food-poisoning is the only likely source of confusion. This can usually be excluded without difficulty, however, by the history of the outbreak and the absence of a likely vehicle for all the children concerned. In the sporadic case, on the other hand, a diagnosis of winter vomiting disease is clearly perilous until other, more serious conditions have been ruled out. In children vomiting may herald the onset of acute fevers or of a surgical emergency, while in adults surgical emergencies, intracranial lesions, or myocardial infarction must be considered.

Apart from outbreaks at schools, most cases of winter vomiting disease are probably not seen by doctors, and their rapid recovery makes treatment unnecessary. In a few cases, however, serious dehydration may require energetic treatment. Deaths in elderly patients with vomiting and diarrhoea were reported in a Cornish outbreak, ${ }^{11}$ but otherwise the disease is almost invariably benign.

Laboratory study of this condition has not been rewarding. In three children concerned in the Sheffield hospital outbreak" examination of the cerebrospinal fluid showed a pleocytosis, underlining the commonly held view that winter vomiting disease is essentially a disease of the central nervous system. Attempts to isolate a virus from patients have not yet succeeded.

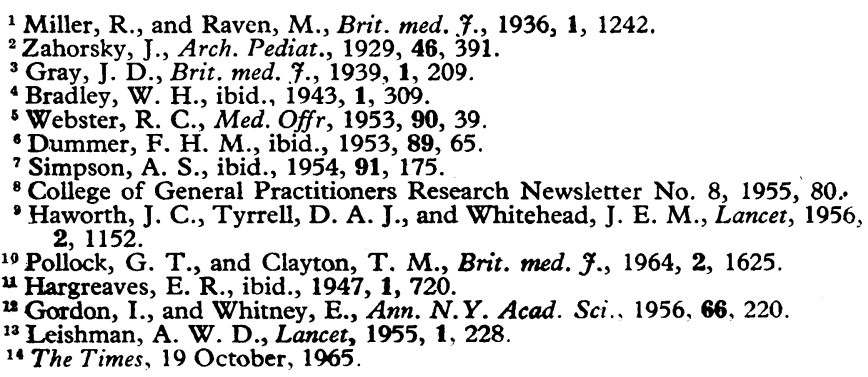

\section{Fatal Chicken-pox}

In one small village during a Christmas holiday two children died from complications of chicken-pox. One had encephalitis and the other developed the haemorrhagic form of the disease. Drs. A. W. Blair, W. M. Jamieson, and G. H. Smith report the cases at page 981 of the B.M.F. this week. Fortunately complications of chicken-pox are rare: In one isolation hospital, serving a population of a million persons, 12 cases of varicella with complications were admitted in 10 years,' half being in patients over the age of 20 . In England and Wales as a whole about 20 deaths a year are recorded."

Pneumonia may develop when the virus invades the lung ; usually the cutaneous rash is widespread and many vesicles are seen in the mouth and pharynx. The patient experiences a painful cough, and sputum may be blood-streaked. $X$-ray changes consist of nodular shadows, which, like the skin rash, change quickly, new ones appearing while earlier ones resolve. $^{3}$ Antibiotics naturally will not overcome the primary infection but may prevent secondary bacterial invasion.

Haemorrhagic signs are perhaps the least common of the serious complications but carry a fairly high mortality. The typical features usually develop within four days of the appearance of the rash. The vesicles take on a haemorrhagic form and there may be extensive purpura. Haemarthroses and sanguineous effusions may be found. The platelet count is usually diminished and the bleeding and clotting times may be altered. Prompt treatment may be needed with blood transfusions and possibly the administration of fibrinogen and platelets.

Neurological complications are associated with many viral infections. They may be caused by direct invasion of the primary virus, activation of another virus latent in the patient, or an allergic response in the nervous system. The exact cause of varicella encephalitis is not yet known. The incidence is probably less than 1 in 10,000 cases and the case mortality about 1 in 10 , but some survivors may suffer permanent sequelae. Symptoms of cerebral damage may precede the rash by three or four days. Most commonly they appear early in the second week, but occasionally are delayed until the end of the third week. As with other forms of encephalitis, the clinical manifestations are variable, and it is possible that electroencephalographic records would show changes in patients with minimal symptoms that pass almost unnoticed. The onset may be gradual or rapid, and almost invariably headache, drowsiness, and a little vomiting are the first warnings of trouble. Coma will develop in about one-fifth of the patients, and in about the same proportion muscular twitching or convulsions will be seen. ${ }^{4}$ Sometimes there is rapid deterioration with the appearance of signs indicating serious and possibly irreversible damage to the brain. In a few cases the symptoms are almost entirely confined to cerebellar dysfunction, an interesting account of which was recently given

\footnotetext{
1 Rotem, C. E., Brit. med. 7., 1961, 1, 944.

2 The Registrar-General's Statistical Review of England and Wales for the Year 1963, Part 1, Tables, Medical. H.M.S.O. 1965.

3 Tan, D. Y. M., Kaufman, S. A., and Levene, G., Amer. 7. Roentgenol., 1956, 76, 527.

4 Miller, H. G., Stanton, J. B., and Gibbons, J. L., Quart. F. Med., 1956, $25,427$.

- Gatley, M. S., Practitioner, 1965, 195, 357.

- Hunter, W. B., Lancet, 1875, 1, 45.

' Jenkins, R. B., Amer. F. Dis. Child., 1965, 110, 137.

Brit. med. F., 1957, 1, 511.
} 
by $M$. S. Gatley. ${ }^{5}$ His patient was not unduly drowsy, nor were any convulsive movements seen, and management was safely and effectively conducted at home.

In 1875 William B. Hunter, ${ }^{6}$ of Matlock, described a 3-year-old boy who started convulsions on the day after he developed the rash of chicken-pox. They were attributed to " incipient cerebral meningitis," and in elegant language the author describes the application of warm packs to the trunk and extremities, cold cloths to the head, and a cambric handkerchief to the face. The child recovered. More sophisticated forms of therapy are now available, and Ramon Jenkins, ${ }^{7}$ of the Moses Cone Hospital in North Carolina, has recently given a detailed description of a case of varicella encephalopathy in which, despite decerebrate rigidity with fixed dilated pupils and hyperventilation of a central type, intensive therapy was followed by an unexpected and gratifying recovery. On the assumption that cerebral oedema might be partly responsible for the severity of the symptoms intravenous urea was administered and was followed by some improvement within four hours. Treatment also included hypothermia, diphenylhydantoin, and dexamethasone. In $1957^{\circ}$ we drew attention to reports suggesting that varicella in patients receiving steroid therapy might take a serious and possibly fatal form. The problem posed by these reports is still unsolved and conflicting advice is freely available. Whether steroids are used or not varicella encephalitis merits prompt treatment, certainly with anticonvulsant drugs (started if possible before convulsions occur), and if the patient is in coma the administration of intravenous urea and hypothermia should be considered.

The family doctor does not usually regard chicken-pox as a problem calling for an urgent visit, but if the patient has a headache and is drowsy, or shows signs of bleeding, prompt attention may be needed. It is to be hoped that parents will continue to regard chicken-pox as a minor ailment only to be feared if it coincides with a school examination or a longawaited family holiday.

\section{Defibrination Syndrome}

If the body's tissue materials escape into the blood they may cause massive coagulation or fibrinolysis, enough to reduce the concentration of plasma fibrinogen suddenly. Reduction of fibrinogen in this way results in the acute defibrination syndrome, a failure of the blood to clot associated with severe shock and haemorrhage.

Usually intravascular coagulation predominates over fibrinolysis, and fibrinogen is lost by being deposited as fibrin at the sites of tissue damage and often throughout the vascular system. Defibrination of this type may sometimes complicate pregnancy when the patient has suffered amniotic-fluid embolism or premature separation of the placenta, as in cases of abruptio placentae, accidental haemorrhage, or placenta praevia. It may also happen during any major surgical procedure, especially thoracic operations and those entailing an extracorporeal circulation, or as a result of incompatible blood transfusion or the bite of certain venomous snakes. A secondary increase of fibrinolysis may set in as a protective response which releases enzymes to dissolve the deposited fibrin. ${ }^{1}$ These enzymes do not attack fibrinogen unless the plasma inhibitors which normally protect it are overwhelmed. They may be when the materials escaping from the tissues are rich in fibrinolytic activators-for example, in some patients with disseminated cancer of the prostate. Fibrinolysis may also increase as a direct result of fibrinolytic enzymes given therapeutically to dissolve thrombi.

In the blood certain signs are common to the defibrination syndrome caused by either coagulation or fibrinolysis. In both there is a reduction of fibrinogen and other clotting factors, especially factor V and factor VIII. Anticoagulants which impede the formation of fibrin from any remaining fibrinogen may also appear, either endogenous heparin, released in response to intravascular coagulation, ${ }^{2}$ or the polypeptide fragments produced by fibrinolysis. In cases due to intravascular coagulation there is a tendency to thrombocytopenia. A feature distinguishing those with massive fibrinolysis is a profound fall in the concentration of plasminogen in the blood owing to its sudden conversion to the enzyme plasmin. ${ }^{3}$

When unexplained bleeding develops in association with any clinical condition in which defibrination is a possibility prompt diagnosis is essential if treatment is to prevent uncontrollable haemorrhage. Though the defects in the clotting mechanism are likely to be multiple, ${ }^{4}$ the diagnosis can be established by showing there is a low concentration of fibrinogen in the blood. Many biochemical methods take too long to do this, but rapid screening tests have been described. ${ }^{56}$ They provide an estimate of fibrinogen from the action of thrombin on a sample of citrated blood from the patient. Important changes could be missed by relying on the simple whole-blood clotting-time alone, although severe defibrination is unlikely if the blood clots firmly within five minutes. Immunological or precipitation methods for measuring total fibrinogen may also give a false sense of security if the formation of fibrin is blocked by an anticoagulant; thrombin tests take this into account and also allow preliminary assessment of the extent of fibrinolysis.

Deficiency of fibrinogen is sometimes an incidental finding, but for a patient who is either shocked or bleeding urgent replacement of the deficit is essential. If the critical concentration of fibrinogen to ensure haemostasis is taken as 100 $\mathrm{mg} . / 100 \mathrm{ml}$. of plasma, then the average adult needs at least $3 \mathrm{~g}$. of fibrinogen in the blood as a whole. Each $500 \mathrm{ml}$. of blood contains less than $1 \mathrm{~g}$. of fibrinogen, and if losses are continuing it may be impossible to transfuse blood at a rate sufficient to meet this need unless a supplementary source of fibrinogen is used. One litre of plasma provides $3 \mathrm{~g}$. and may suffice if it can be transfused within half an hour either as fresh or fresh frozen plasma, which will also tend to correct other clotting-factor deficiencies, or in smaller volumes as double- or triple-strength reconstituted dried plasma. The replacement therapy must be controlled with repeated blood tests, and if larger amounts of fibrinogen are needed, as they often may be, purified concentrates can be given in small volumes.

Rapid clinical improvement usually follows when an adequate concentration of fibrinogen in the plasma is

\footnotetext{
Fletcher, A. P., Alkjaersig, N., and Sherry, S., Amer. F. Med., 1962, 33, 738 .

2 Hardaway, R. M., and Drake, D. C., Ann. Surg., 1963, 157, 39.

Flute, P. T., Proc. roy. Soc. Med., 1964, 57, 603.

Brit. med. F., 1960, 1, 789.

Ingram, G. I. C., f. clin. Path., 1961, 14, 356.

Hardisty, R. M., Ingram, G. I. C., and Sharp, A. A., Broadsheet 48 (New Series). Association of Clinical Pathologists, 1964.

Brit. med. F., 1965, 1, 704.

s Naeye, R. L., Blood, 1962, 19, 694.
} 